\title{
Higher Order Eigenpair Perturbations
}

\author{
M. S. Eldred* \\ University of Michigan, Ann Arbor, Michigan 48109 \\ P. B. Lerner† \\ Los Alamos National Laboratory, Los Alamos, New Mexico 87544 \\ and \\ W. J. Anderson $\ddagger$ \\ University of Michigan, Ann Arbor, Michigan 48109
}

\begin{abstract}
A matrix method is presented for the exact calculation of large eigenpair perturbations arising from design variable changes. The method is "forward analysis" in the sense that design variable changes are known and eigenpair perturbations are computed. Competing methods for forward analysis include reanalysis and firstorder approximate methods. First-order methods are well known for their ability to calculate small eigenpair changes but are inadequate when large changes are required. In this paper, a new method of forward analysis is presented that takes into account all orders of the perturbation expansion. A benchmark cantilever beam will be analyzed with the new method for large perturbations in design variables. The method will be valuable for fast reanalysis and for identification and model correlation studies where sizeable differences exist between a baseline model and an objective model.
\end{abstract}

\section{Nomenclature}

$c_{i} \quad=$ weighting factor determining the contribution of the homogeneous solution to the total solution for $\{\Delta \phi\}_{i}$

$[D]_{i}=$ coefficient matrix in solution for $\{\Delta \phi\}_{i}$; equals $[K]-\lambda_{i}[M]$

$\left\{F^{l}\right\}_{i}=$ static pseudoload vector appearing in linearized $\{\Delta \phi\}_{i}$ calculation, $=\left(\Delta \lambda_{i}\left[M^{0}\right]+\lambda_{i}^{0}[\Delta M]\right.$

$-[\Delta K])\left\{\phi^{0}\right\}_{i}$; orthogonal to $\left\{\phi^{0}\right\}_{i}$

$\left\{F^{n l}\right\}_{i}=$ static pseudoload vector appearing in nonlinear $\{\Delta \phi\}_{i}$ calculation, $=\left(\Delta \lambda_{i}\left[M^{1}\right]+\lambda_{i}^{0}[\Delta M]\right.$

$-[\Delta K])\left\{\phi^{0}\right\}_{i}$; orthogonal to $\left\{\phi^{1}\right\}_{i}$

$[K]=$ stiffness matrix, symmetric

$[M] \quad=$ mass matrix, symmetric

$\Delta \quad=$ perturbation symbol denoting exact change from the baseline

$\lambda_{i}=i$ th eigenvalue (circular frequency squared)

$\{\phi\}_{i}=i$ th eigenvector (i.e., mode shape of structure); solution to $[K]\{\phi\}_{i}=\lambda_{i}[M]\{\phi\}_{i}$; normalized with respect to appropriate $[M]$

Superscripts

$0 \quad=$ baseline values

$1=$ perturbed values resultant from design variable change, e.g., ()$^{1}=()^{0}+\Delta()$

\section{Introduction}

$\mathbf{C}$ URRENT methods for optimization in structural dynamics use derivatives with respect to design variables. When numerically calculating these derivatives, one commonly uses perturbations of $2 \%$ or less in the design variables. With design changes this small, the eigenpair perturbations calculated are small, and the resulting sensitivities are derivatives at the baseline configuration. Currently popular methods of cal-

Received May 20, 1991; revision received Oct. 23, 1991; accepted for publication Oct. 28, 1991. Copyright (C) 1992 by the Authors. Published by the American Institute of Aeronautics and Astronautics, Inc., with permission.

* Graduate Research Assistant, Department of Aerospace Engineering. Student Member AIAA.

†Physicist, P.O. Box 1663, MS K723.

$\ddagger$ Professor of Aerospace Engineering, Department of Aerospace Engineering. Senior Member AIAA. culating eigenpair sensitivities all compute the derivative values, either directly through differentiating the eigenproblem statement or indirectly through calculating small change in first-order perturbation equations (having dropped higher order terms). It is important to realize that the perturbation equations of first order in $\Delta$ are the same as the derivative equations.

\section{Literature Survey and Research Impetus}

Fox and Kapoor's method ${ }^{1}$ differentiates the eigenproblem equation and calculates derivatives by a modal superposition method. It usually requires a full set of modes to obtain exact derivatives (counterexample: tip mass as design variable in Ref. 2). Wang's method ${ }^{3}$ modifies Fox and Kapoor's method for the case of a truncated set of modes through the use of a residual static mode. Nelson's method ${ }^{4}$ calculates derivatives through removing the singularity in the matrix equation and solving directly. It needs information associated with the $i$ th mode only, so the issue of using a truncated modal set is not important. High's method ${ }^{5}$ iteratively computes small eigenpair perturbations and, for large systems, is faster than direct calculation. This method has been implemented in MSC/ NASTRAN, but the iterations are divergent for anything other than small change. ${ }^{6}$ Surveys and comparisons of these and other small change methods are readily available in the literature. . $^{2,7}$

The work presented here departs from small change ideas and calculates large changes in the eigenpairs by handling the nonlinear nature of the perturbation equations. Kim et al. ${ }^{8}$ and Hoff et al. ${ }^{9}$ have explored similar directions. Another recent paper developed independently by $\mathrm{Kim}^{10}$ is very similar in spirit to the current work, but the solution procedure is different and a first-order normalization is used. As presented, this paper's method is most easily characterized as fast reanalysis, since the changes calculated are exact. If large changes are present, dividing the eigenpair perturbations by the change in the design variable yields secant sensitivity values instead of tangent derivative values. Thus, the proper term might be eigenpair secant sensitivities rather than eigenpair derivatives.

The retention of all terms in the expansion of the governing equations (eigenproblem, normalization) is required to study large changes in the eigenpairs resulting from large changes in design variables. Consideration of large change is unnecessary in the current applications of eigenpair derivative technology 
known to the authors. Potential use can be foreseen in the areas of model correlation and system identification in which large exact changes would be more desirable than small approximate changes. Although these are generally "inverse analysis" (eigenpair perturbations known, design variable perturbations desired), the forward analysis would be necessary in a predictor/corrector algorithm. ${ }^{9}$ As an example of large change, consider damage to a large space truss in which an element has been destroyed. This requires a $100 \%$ reduction in stiffness for that element (a redundant structure is required). The small change methods referenced previously would fail in calculating meaningful eigenpair derivatives since they are generally restricted to changes of only a few percent. Another potential use is optimization work in which criteria other than frequencies and mode shapes drive the design and in which frequencies and mode shapes are allowed to drift (or are inactive constraints). When this is the case, large changes in frequencies and modes may occur on each redesign iteration, and large change algorithms would be needed to track them.

\section{Repeated Eigenvalues}

The subject of repeated eigenvalues has recently received a great deal of attention. Modal superposition techniques such as Fox and Kapoor's method are particularly susceptible to problems when multiplicity is present. The work of Kim and Wallerstein ${ }^{11}$ is most easily applied to this paper's technology. They employ an orthogonal transformation to generate a set of complementary mode shapes. All baseline mode shapes are required. The only modification necessary for the authors' method to handle repeated eigenvalues, according to Kim, is the use of these complementary mode shapes in place of the baseline mode shapes.

\section{Equation Derivations}

Turning attention to the derivation of the governing equations, consider the classical, linear, structural eigenproblem:

$$
\left[K^{0}\right]\left\{\phi^{0}\right\}_{i}=\lambda_{i}^{0}\left[M^{0}\right]\left\{\phi^{0}\right\}_{i}
$$

When the system is perturbed from the baseline through mass and stiffness changes, the new statement of dynamic equilibrium is

$$
\left[K^{1}\right]\left\{\phi^{1}\right\}_{i}=\lambda_{i}^{1}\left[M^{1}\right]\left\{\phi^{1}\right\}_{i}
$$

or in expanded form

$$
\begin{aligned}
& \left(\left[K^{0}\right]+[\Delta K]\right)\left(\left\{\phi^{0}\right\}_{i}+\{\Delta \phi\}_{i}\right) \\
& \quad=\left(\lambda_{i}^{0}+\Delta \lambda_{i}\right)\left(\left[M^{0}\right]+[\Delta M]\right)\left(\left\{\phi^{0}\right\}_{i}+\{\Delta \phi\}_{i}\right)
\end{aligned}
$$

Expanding, canceling the baseline solution, and regrouping yields

$$
\begin{gathered}
\left(\left[K^{0}\right]-\lambda_{i}^{0}\left[M^{0}\right]\right)\{\Delta \phi\}_{i}+\left([\Delta K]-\lambda_{i}^{0}[\Delta M]\right)\left(\left\{\phi^{0}\right\}_{i}+\{\Delta \phi\}_{i}\right) \\
\quad=\Delta \lambda_{i}\left(\left[M^{0}\right]+[\Delta M]\right)\left(\left\{\phi^{0}\right\}_{i}+\{\Delta \phi\}_{i}\right)
\end{gathered}
$$

Premultiplying by $\left\{\phi^{0}\right\}_{i}^{T}$ and canceling the baseline solution again (due to the symmetry of $\left[M^{0}\right]$ and $\left[K^{0}\right]$ ) yields

$$
\begin{aligned}
& \left\{\phi^{0}\right\}_{i}^{T}\left([\Delta K]-\lambda_{i}^{0}[\Delta M]\right)\left(\left\{\phi^{0}\right\}_{i}+\{\Delta \phi\}_{i}\right) \\
& \quad=\Delta \lambda_{i}\left\{\phi^{0}\right\}_{i}^{T}\left(\left[M^{0}\right]+[\Delta M]\right)\left(\left\{\phi^{0}\right\}_{i}+\{\Delta \phi\}_{i}\right)
\end{aligned}
$$

\section{First-Order System of Equations}

Popular derivative solutions and first-order approximations (specifically Fox and Kapoor's method, ${ }^{1}$ Wang's method, ${ }^{3}$ Nelson's method, ${ }^{4}$ and High's method ${ }^{5}$ ) all solve equations derivable by dropping the terms of higher order in $\Delta$. When this is done, Eq. (5) becomes

$$
\left\{\phi^{0}\right\}_{i}^{T}\left([\Delta K]-\lambda_{i}^{0}[\Delta M]\right)\left\{\phi^{0}\right\}_{i} \cong \Delta \lambda_{i}\left\{\phi^{0}\right\}_{i}^{T}\left[M^{0}\right]\left\{\phi^{0}\right\}_{i}
$$

Employing the normalization condition $\left\{\phi^{0}\right\}_{i}^{T}\left[M^{0}\right]\left\{\phi^{0}\right\}_{i}=1$, one obtains the following uncoupled equation for $\Delta \lambda_{i}$ :

$$
\Delta \lambda_{i} \cong\left\{\phi^{0}\right\}_{i}^{T}\left([\Delta K]-\lambda_{i}^{0}[\Delta M]\right)\left\{\phi^{0}\right\}_{i}
$$

In a like manner, one drops the higher order terms from Eq. (4) and solves for $\{\Delta \phi\}_{i}$ to obtain the other important firstorder formula:

$$
\left(\left[K^{0}\right]-\lambda_{i}^{0}\left[M^{0}\right]\right)\{\Delta \phi\}_{i} \cong\left(\Delta \lambda_{i}\left[M^{0}\right]+\lambda_{i}^{0}[\Delta M]-[\Delta K]\right)\left\{\phi^{0}\right\}_{i}
$$

This formula can be written as

$$
\left[D^{0}\right]_{i}\{\Delta \phi\}_{i} \cong\left\{F^{l}\right\}_{i}
$$

where

$$
\left[D^{0}\right]_{i} \equiv\left[K^{0}\right]-\lambda_{i}^{0}\left[M^{0}\right]
$$

is singular and

$$
\left\{F^{\prime}\right\}_{i} \equiv\left(\Delta \lambda_{i}\left[M^{0}\right]+\lambda_{i}^{0}[\Delta M]-[\Delta K]\right)\left\{\phi^{0}\right\}_{i}
$$

can be viewed as a static pseudoload. This equation corresponds to the pathological Fredholm alternative in which the coefficient matrix is singular and there is a nonzero load. Such equations cannot be solved in general. This equation is solvable, however, since it is "consistent," i.e., $\left\{F^{l}\right\}_{i}$ is orthogonal to $\left\{\phi^{0}\right\}_{i}$. (Note: the fact that this first-order system is consistent must be viewed as fortunate.)

To prove the consistency of the linearized problem, one premultiplies $\left\{F^{\prime}\right\}$ by the transpose of the baseline eigenvector:

$$
\begin{aligned}
& \left\{\phi^{0}\right\}_{i}^{T}\left\{F^{\prime}\right\}_{i}=\Delta \lambda_{i}\left\{\phi^{0}\right\}_{i}^{T}\left[M^{0}\right]\left\{\phi^{0}\right\}_{i}+\lambda_{i}^{0}\left\{\phi^{0}\right\}_{i}^{T}[\Delta M]\left\{\phi^{0}\right\}_{i} \\
& -\left\{\phi^{0}\right\}_{i}^{T}[\Delta K]\left\{\phi^{0}\right\}_{i}
\end{aligned}
$$

Substituting the first-order equation for $\Delta \lambda_{i}$ [Eq. (7)] cancels all terms on the right-hand side:

$$
\begin{gathered}
\left\{\phi^{0}\right\}_{i}^{T}\left\{F^{l}\right\}_{i}=\left\{\phi^{0}\right\}_{i}^{T}[\Delta K]\left\{\phi^{0}\right\}_{i}-\lambda_{i}^{0}\left\{\phi^{0}\right\}_{i}^{T}[\Delta M]\left\{\phi^{0}\right\}_{i} \\
+\lambda_{i}^{0}\left\{\phi^{0}\right\}_{i}^{T}[\Delta M]\left\{\phi^{0}\right\}_{i}-\left\{\phi^{0}\right\}_{i}^{T}[\Delta K]\left\{\phi^{0}\right\}_{i} \\
=0
\end{gathered}
$$

\section{Nonlinear System of Equations}

The focus of this paper will be on the nonlinear perturbation equations and the retention of all higher order terms. The first nonlinear formula is obtained by solving Eq. (5) for $\Delta \lambda_{i}$ :

$$
\Delta \lambda_{i}=\frac{\left\{\phi^{0}\right\}_{i}^{T}\left([\Delta K]-\lambda_{i}^{0}[\Delta M]\right)\left\{\phi^{1}\right\}_{i}}{\left\{\phi^{0}\right\}_{i}^{T}\left[M^{1}\right]\left\{\phi^{1}\right\}_{i}}
$$

The second nonlinear equation is derived by rearranging the terms of Eq. (4) so as to collect all $\{\Delta \phi\}_{i}$ terms on the left-hand side:

$$
\begin{aligned}
& \left(\left[K^{0}\right]-\lambda_{i}^{0}\left[M^{0}\right]\right)\{\Delta \phi\}_{i}+\left([\Delta K]-\lambda_{i}^{0}[\Delta M]\right)\{\Delta \phi\}_{i} \\
& -\Delta \lambda_{i}\left(\left[M^{0}\right]+[\Delta M]\right)\{\Delta \phi\}_{i}=\Delta \lambda_{i}\left(\left[M^{0}\right]+[\Delta M]\right)\left\{\phi^{0}\right\}_{i} \\
& -\left([\Delta K]-\lambda_{i}^{0}[\Delta M]\right)\left\{\phi^{0}\right\}_{i}
\end{aligned}
$$


Combining terms yields the relation

$$
\left(\left[K^{1}\right]-\lambda_{i}^{1}\left[M^{1}\right]\right)\{\Delta \phi\}_{i}=\left(\Delta \lambda_{i}\left[M^{1}\right]+\lambda_{i}^{0}[\Delta M]-[\Delta K]\right)\left\{\phi^{0}\right\}_{i}
$$

This equation, as its linearized counterpart, can be written

$$
\left[D^{1}\right]_{i}\{\Delta \phi\}_{i}=\left\{F^{n I}\right\}_{i}
$$

where

$$
\left[D^{1}\right]_{i} \equiv\left[K^{1}\right]-\lambda_{i}^{1}\left[M^{1}\right]
$$

is singular and

$$
\left\{F^{n l}\right\}_{i} \equiv\left(\Delta \lambda_{i}\left[M^{1}\right]+\lambda_{i}^{0}[\Delta M]-[\Delta K]\right)\left\{\phi^{0}\right\}_{i}
$$

is a static pseudoload. This equation is also consistent, though this time the pseudoload vector is orthogonal to $\left\{\phi^{1}\right\}_{i}$ instead of $\left\{\phi^{0}\right\}_{i}$.

The proof of the consistency for the nonlinear problem can be performed by premultiplying $\left\{F^{n l}\right\}_{i}$ by the transpose of the perturbed eigenvector. The same cancellation of terms occurs as in the linear case after substituting the exact equation for $\Delta \lambda_{i}\left[\mathrm{Eq}\right.$. (14)] and employing the symmetry of $\left[M^{1}\right],[\Delta M]$, and $[\Delta K]$.

Equations (14) and (17) look quite similar in form to Eqs. (7) and (9). Their solution methodology is very different, however. Equations (14) and (17) are coupled since $\Delta \lambda_{i}$ is dependent on $\left\{\phi^{1}\right\}_{i}$ and $\{\Delta \phi\}_{i}$ is dependent on $\lambda_{i}^{1}$. Simultaneous solution seems impossible. Thus, solution of this system must be done iteratively. The first-order system, on the other hand, may be solved directly since $\Delta \lambda_{i}$ depends only on known quantities in Eq. (7).

\section{Theoretical Development}

Solution for the exact eigenpair changes is now a problem of correctly solving Eqs. (14) and (17). This is not easy due to the coupling of the equations and to the inherent instability of the problem. The proposed method of attack is to find an initial guess for $\Delta \lambda_{i}$ and then to iterate Eqs. (14) and (17).

There are four important points in the mathematics of the problem that must be incorporated in the iteration scheme. These points are 1) the switching of solution logic as $\left[D^{1}\right]_{i}$ is driven singular, 2) the deletion of the spurious solution for $\left.\{\Delta \phi\}_{i}, 3\right)$ the proper management of the homogeneous and particular solutions for $\{\Delta \phi\}_{i}$, and 4$)$ the calculation of the particular solution.

\section{Point 1: Switching of Solution Logic}

The singularity of the matrix $\left[D^{1}\right]_{i}$ is very important and is dependent on the accuracy of the $\lambda_{i}^{1}$ estimate at a particular iteration. For a nonexact estimate, the $\left[D^{1}\right]_{i}$ matrix is nonsingular and can be inverted for an estimate of $\{\Delta \phi\}_{i}$. When the $\lambda_{i}^{1}$ estimate has converged, the matrix is singular and what is herein called a modified Nelson's method is used to find the particular solution, which is in turn used to find $\{\Delta \phi\}_{i}$.

On the starting iteration, the $\lambda_{i}^{1}$ estimate is relatively poor and $\left[D^{1}\right]_{i}$ is nonsingular. There are no homogeneous and particular solutions to manage, and $\{\Delta \phi\}_{i}$ is found from standard matrix decomposition techniques. This solution for $\{\Delta \phi\}_{i}$ will be heavily corrupted by its spurious solution $\left(-\left\{\phi^{0}\right\}_{i}\right)$ since the system is nonsingular (see point 2$)$. On subsequent iterations, the $\left[D^{1}\right]_{i}$ matrix will be treated as if singular, and the modified Neison's method is used to find the particular solution (see points 3 and 4).

\section{Point 2: Deletion of Spurious Solution}

The spurious solution for $\{\Delta \phi\}_{i}$ can be seen in Eq. (3), where it is evident that $\{\Delta \phi\}_{i}=-\left\{\phi^{0}\right\}_{i}$ trivially satisfies the equality. When $\left[D^{1}\right]_{i}$ is only nearly singular due to an approximate $\lambda_{i}^{l}$, the solution for $\{\Delta \phi\}_{i}$ is driven strongly toward this spurious solution. It is only when $\left[D^{1}\right]_{i}$ is exactly singular (convergent $\lambda_{i}^{l}$ ) that a meaningful $\{\Delta \phi\}_{i}$ is achievable.

The easiest way (and so far the best way) to delete the unwanted spurious solution is to simply add $\left\{\phi^{0}\right\}_{i}$ to the corrupted $\{\Delta \phi\}_{i}$ solution and scale the result to satisfy perturbed mass normalization (see Ref. 6 for discussion of mass normalization schemes). An alternative method would be to orthogonalize the $\{\Delta \phi\}_{i}$ solution to $\left\{\phi^{0}\right\}_{i}$ by using a GramSchmidt orthogonalization process, but this has not been as effective.

The deletion of the spurious solution has only been necessary on the starting iteration in which $\{\Delta \phi\}_{i}$ is found from standard matrix decomposition techniques (nonsingular $\left[D^{1}\right]_{i}$ ). It is, however, always an option if convergence difficulties are encountered on subsequent iterations.

\section{Point 3: Homogeneous and Particular Solutions}

As for a nonhomogeneous differential equation, the total solution for $\{\Delta \phi\}_{i}$ is made up of homogeneous and particular solutions. When $\left[D^{1}\right]_{i}$ is exactly singular, $\left\{\phi^{1}\right\}_{i}$ is a homogeneous solution for $\{\Delta \phi\}_{i}$ in Eq. (17) since $\left[D^{1}\right]_{i}\left\{\phi^{1}\right\}_{i}=\{0\}$. The corresponding particular solution will be called $\{V\}_{i}$. The total solution for $\{\Delta \phi\}_{i}$ is then a sum of $\{V\}_{i}$ and a weighted $\left\{\phi^{1}\right\}_{i}$. The weighting factor $c_{i}$ is introduced because the scaling of the homogeneous solution is initially indeterminate:

For singular $\left[D^{1}\right]_{i}$ :

$$
\{\Delta \phi\}_{i}=c_{i}\left\{\phi^{1}\right\}_{i}+\{V\}_{i}
$$

The following derivation will employ conditions of mass normalization to determine the weighting factor $c_{i}$. Equation (20) must be altered since $\left\{\phi^{1}\right\}_{i}$ is unknown:

$$
\begin{gathered}
\{\Delta \phi\}_{i}=c_{i}\left(\left\{\phi^{0}\right\}_{i}+\{\Delta \phi\}_{i}\right)+\{V\}_{i} \\
\left(1-c_{i}\right)\{\Delta \phi\}_{i}=c_{i}\left\{\phi^{0}\right\}_{i}+\{V\}_{i} \\
\{\Delta \phi\}_{i}=\frac{c_{i}}{1-c_{i}}\left\{\phi^{0}\right\}_{i}+\frac{1}{1-c_{i}}\{V\}_{i}
\end{gathered}
$$

A concern at this point is whether or not $c_{i}$ can attain the value of unity, since the solution of Eq. (23) would be undefined. In practice, $c_{i}$ is much smaller than unity, meaning that the contribution of the homogeneous solution to the total solution is small. This fact can be shown with a heuristic proof by contradiction. Letting $c_{i}=1$ in Eq. (21) cancels the $\{\Delta \phi\}_{i}$ terms, leaving the particular solution equal to the spurious solution $\left(\{V\}_{i}=-\left(\phi^{0}\right\}_{i}\right)$. But the spurious solution is deleted in the starting iteration, and the particular solution should not be corrupted with the spurious solution. Therefore, $c_{i}$ must be different than unity.

The mass normalization criteria for $\left\{\phi^{0}\right\}_{i}$ and $\left\{\phi^{1}\right\}_{i}$ are

$$
\begin{aligned}
& \left\{\phi^{1}\right\}_{i}^{T}\left[M^{1}\right]\left\{\phi^{1}\right\}_{i}=1 \\
& \left\{\phi^{0}\right\}_{i}^{T}\left[M^{0}\right]\left\{\phi^{0}\right\}_{i}=1
\end{aligned}
$$

Subtracting the two equations and expanding yields

$$
\begin{aligned}
& \left.\left(\left\{\phi^{0}\right\}_{i}+\{\Delta \phi\}_{i}\right)^{T}\left(\left[M^{0}\right]\right)+[\Delta M]\right)\left(\left\{\phi^{0}\right\}_{i}+\{\Delta \phi\}_{i}\right) \\
& -\left\{\phi^{0}\right\}_{i}^{T}\left[M^{0}\right]\left\{\phi^{0}\right\}_{i}=0
\end{aligned}
$$

$$
\begin{gathered}
2\{\Delta \phi\}_{i}^{T}\left[M^{0}\right]\left\{\phi^{0}\right\}_{i}+\{\Delta \phi\}_{i}^{T}\left[M^{0}\right]\{\Delta \phi\}_{i}+\left\{\phi^{0}\right\}_{i}^{T}[\Delta M]\left\{\phi^{0}\right\}_{i} \\
+2\{\Delta \phi\}_{i}^{T}[\Delta M]\left\{\phi^{0}\right\}_{i}+\{\Delta \phi\}_{i}^{T}[\Delta M]\{\Delta \phi\}_{i}=0
\end{gathered}
$$


Combining terms gives the exact normalization equation for $\{\Delta \phi\}_{i}$ :

$2\{\Delta \phi\}_{i}^{T}\left[M^{1}\right]\left\{\phi^{0}\right\}_{i}+\{\Delta \phi\}_{i}^{T}\left[M^{1}\right]\{\Delta \phi\}_{i}=-\left\{\phi^{0}\right\}_{i}^{T}[\Delta M]\left\{\phi^{0}\right\}_{i}$

Substituting Eq. (23) into Eq. (28) for $\{\Delta \phi\}_{i}$ gives, after some algebraic manipulation,

$$
\xi_{i}+\left(2+\xi_{i}\right) c_{i}+c_{i}^{2}-c_{i}^{3}=0
$$

where

$$
\xi_{i}=\left\{\phi^{0}\right\}_{i}^{T}[\Delta M]\left\{\phi^{0}\right\}_{i}+2\left\{\phi^{0}\right\}_{i}^{T}\left[M^{1}\right]\{V\}_{i}+\{V\}_{i}^{T}\left[M^{1}\right](V\}_{i}
$$

Equation (29) has roots

$$
c_{i}=-1, \quad 1 \pm \sqrt{1+\xi_{i}}
$$

The proper root is the one for which $\left|c_{i}\right| \ll 1$, i.e.

$$
c_{i}=1-\sqrt{1+\xi_{i}}
$$

\section{Point 4: Calculation of Particular Solution}

The only remaining problem is the most difficult one, the calculation of the particular solution $\{V\}_{i}$. This step is not needed on the starting iteration, when $\left[D^{1}\right]_{i}$ is nonsingular.

A brief review of Nelson's method ${ }^{4}$ is necessary at this point. The objective is to solve for the particular solution $\{V\}_{i}$ of Eq. (9) by directly removing the singularity in $\left[D^{0}\right]_{i}$. This is accomplished by "zeroing out" a row and column of $\left[D^{0}\right]_{i}$, putting a 1 at the row and column intersection, and placing a zero in the same row of $\left\{F^{\prime}\right\}_{i}$, effectively enforcing a zero value on the corresponding component of $\{V\}_{i}$. The row and column chosen is essential to the success of the scheme and corresponds to the maximum magnitude component of the $i$ th baseline eigenvector. For example, if the fifth component of $\left\{\phi^{0}\right\}_{i}$ has maximum magnitude, then one puts zeros on the fifth row and column of $\left[D^{0}\right]_{i}$ and the fifth row of $\left\{F^{l}\right\}_{i}$ and

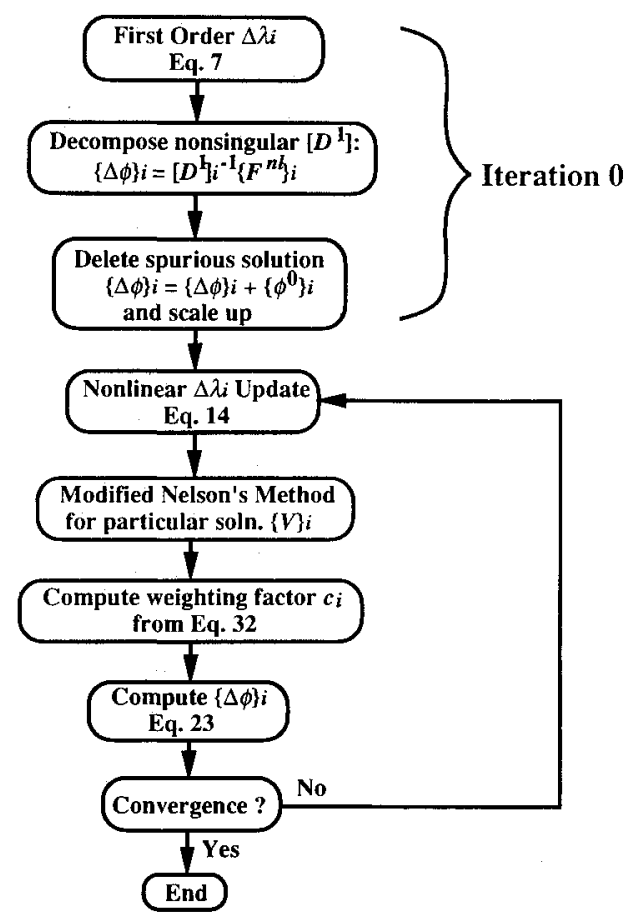

Fig. 1 Solution algorithm. puts a 1 at $D_{55_{i}}^{0}$. This drives the fifth component of $\{V\}_{i}$ to zero in the subsequent matrix decomposition. This method is widely used and is efficient when the coefficient matrix is exactly singular. When the matrix is only nearly singular, however, a modification must be made.

The modified Nelson's method developed here has two differences from the original method. First, one solves for the particular solution of Eq. (17) instead of Eq. (9). This is a trivial point since Nelson's method would work equally well on either equation as long as both coefficient matrices were exactly singular. The second change handles the nonsingularity of $\left[D^{1}\right]_{i}$

Instead of enforcing a zero value on the component of $\{V\}_{i}$, we will prescribe a finite value derived from previous $\{\Delta \phi\}_{i}$ information. On iteration $0,\{\Delta \phi\}_{i}$ was found from a standard matrix decomposition. Thus, we retain more of the physics of the problem if we set the component of $\{V\}_{i}$ equal to the value of the corresponding component of $\{\Delta \phi\}_{i}$ found on iteration 0 . The difference is given in the following equations:

Nelson's method:

$$
V_{* i}=\mathbf{0}
$$

Modified Nelson's method:

$$
V_{* i}=\Delta \phi_{* i} \quad(\text { from iteration } 0)
$$

where the asterisk denotes the component of interest discussed above. Computationally, this makes a monumental difference in the ability of the iterations to converge. Note that the finite change prescribed does not need to be updated since it becomes less important as $\left[D^{1}\right]_{i}$ becomes more singular; that is, it is just a convergence catalyst and does not influence the final answer.

\section{Solution Procedure}

The points addressed earlier each fall neatly into place in the solution algorithm. The algorithm is shown schematically in Fig. 1. The 0th iteration is the starting iteration in which an initial guess on the eigenvalue changes $\left(\Delta \lambda_{i}\right)$ is obtained from Fox and Kapoor's method, and the $\left[D^{1}\right]_{i}$ matrices are decomposed in the standard way (Nelson's method is unnecessary) to get the first estimate on the eigenvector changes $\left(\{\Delta \phi\}_{i}\right)$. The eigenvector change on this starting iteration must have the spurious solution removed by adding $\left\{\phi^{0}\right\}_{i}$ and then must be scaled to satisfy the perturbed mass normalization. Subsequent iterations use the full nonlinear update for $\Delta \lambda_{i}$ [Eq. (14)] and the modified Nelson's method for solving Eq. (17) for its particular solution $\{\boldsymbol{V}\}_{i}$. Removing the spurious solution has not been necessary on these iterations. Equation (32) is then solved for the weighting factor $c_{i}$ and $\{\Delta \phi\}_{i}$ is updated from Eq. (23). Note that whenever an equation calls for a perturbed value (e.g., $\lambda_{i}^{1}$ ), the sum of the baseline and the most current perturbation (e.g., $\lambda_{i}^{0}+\Delta \lambda_{i}$ ) is used.

With the essential equations derived and the solution methodology outlined, the focus will now turn to solving an example problem.

\section{Benchmark Problem: Cantilever Beam \\ Test Problem}

A five-element Euler-Bernoulli cantilever beam model (Fig. 2) is proposed as a benchmark problem due to its simplicity in finite element analysis. The important material constants are $E=2.0684 \times 10^{5} \mathrm{MPa}, \nu=0.3, \rho=7.8334 \times 10^{-9} \mathrm{~N} \mathrm{~s}^{2} / \mathrm{mm}^{4}$. The motion is constrained to allow only $x z$ bending, which leaves a total of 10 degrees of freedom $(z$ displacement and $y$ rotation at each of five nodes). Element stiffness and mass matrices are assembled and used to find the baseline eigenpairs through the use of a simple MATLAB ${ }^{\text {TM }}$ program $^{12}$ (MAT$\mathrm{LAB}^{\mathrm{TM}}$ is a high-level matrix manipulation language that pro- 
Table 1 Design variable scaling sets

\begin{tabular}{cccccccc}
\hline \hline Set Magnitude, \% & DV1, \% & DV2, \% & DV3, \% & DV4, \% & DV5, \% & $\begin{array}{c}\text { Converged } \\
\text { eigenpairs }\end{array}$ \\
\hline 1 & Kim & +19.15 & -2.89 & -9.01 & -6.34 & -8.27 & 10 \\
2 & \pm 20 & +19.15 & -17.89 & +19.01 & -18.34 & -17.27 & 10 \\
3 & \pm 25 & +25 & -24 & +24.5 & -23 & +24.7 & 10 \\
4 & \pm 30 & +28.5 & -29 & +27 & -28 & +30 & 10 \\
5 & \pm 40 & +39.15 & -37.89 & +39.01 & -38.34 & +37.27 & 9 \\
\hline \hline
\end{tabular}

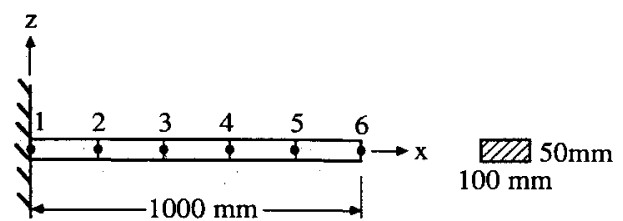

Fig. 2 Cantilever beam modeled with five elements.

vides easy access to the matrix software developed by the LINPACK and EISPACK projects). Upon prescription of design variable changes, one computes new stiffness and mass matrices and Fox and Kapoor's method is performed to get an initial estimate of the eigenvalue perturbations. If the prescribed changes are large, this initial estimate will be in error and the nonlinear iteration scheme described herein must be performed. The aforementioned $100 \%$ reduction in an element's stiffness cannot be tested here since the structure is not redundant.

Several measures have been developed to monitor the progress of the iterations. First, convergence of the eigenvalues is shown through the average of the absolute percent error in each $\lambda_{i}^{1}$, where

$$
\text { absolute percent error }=\left|\frac{\lambda_{i}^{1}-\lambda_{i_{\text {exact }}^{1}}^{1}}{\lambda_{i_{\text {exact }}}^{1}}\right| * 100
$$

and $i$ ranges from 1 to 10 (Table 2 heading: average | $\%$ error $\mid$ in $\left.\lambda_{i}^{1}\right)$. Second, the increasing singularity of $\left[D^{1}\right]_{i}$ is shown through the average condition numbers of these matrices (Table 2 heading: average $\left[D^{1}\right]_{i}$ cond no.). Finally, convergence of the $\left\{\phi^{1}\right\}_{i}$ vectors is monitored through an average of the Euclidean norms of $\left\{\phi^{1}\right\}_{i}-\left\{\phi^{1}\right\}_{i_{\text {exact }}}$ (table heading: average $\|\left\{\phi^{1}\right\}_{i}$ error $\left.\|_{2}\right)$.

Five design variable scaling sets are to be analyzed, each of which perturbs all design variables simultaneously. This is not the typical process for calculating sensitivities, where only one variable is perturbed. Simultaneous perturbations were performed to test the algorithm, since obtaining convergent reșults will obviously be more difficult than for a single design variable perturbation. The five design variable scalings sets are shown in Table 1 and the design variables (abbreviated DV) being scaled are the corresponding beam element heights.

The first set corresponds to case 1 , subcase 3 of the cantilever beam work of Kim et al. ${ }^{8}$ It was chosen to obtain verification of results. The other four design variable scaling sets were chosen arbitrarily to have varying signs and similar magnitude scalings. The last column shows the number of perturbed eigenvectors that converged in the iteration scheme, which will be discussed in the following section.

\section{Results and Discussion}

Table 2 shows the iteration history for design variable scaling set 1 . Improvement of more than an order of magnitude can be seen in all three measures of convergence for each iteration following the first.

To give meaning to these convergence measures, detailed data will be supplied for iteration 3 shown in Table 2 . In Table 3 , the perturbed eigenvalues at iteration 3 are compared with the baseline eigenvalues, the perturbed eigenvalues returned by Fox and Kapoor's modal method (iteration 0), and the exact perturbed eigenvalues.
Table 2 Convergence of iteration-moderate change

\begin{tabular}{lccc}
\hline \hline & $\begin{array}{c}\text { Average } \\
\text { Iter. no. }\end{array}$ & $\begin{array}{c}\text { Average } \\
{\left[\begin{array}{l}\text { error } \mid \text { in } \lambda_{i}^{1} \\
{\left[D^{1}\right]_{i} \text { cond no. }}\end{array}\right.}\end{array}$ & $\begin{array}{c}\text { Average } \\
\left.\| \phi^{1}\right\}_{i} \text { error } \|_{2}\end{array}$ \\
\hline 0 & 3.8161 Fox & $4.2551 \mathrm{e}-06$ & 1.1977 \\
1 & 3.9633 & $4.0960 \mathrm{e}-06$ & 0.8927 \\
2 & 0.1386 & $1.4377 \mathrm{e}-07$ & 0.0352 \\
3 & 0.0055 & $7.6016 \mathrm{e}-09$ & 0.0015 \\
4 & $2.4415 \mathrm{e}-4$ & $4.2493 \mathrm{e}-10$ & $7.3449 \mathrm{e}-5$ \\
5 & $1.1735 \mathrm{e}-5$ & $2.4589 \mathrm{e}-11$ & $3.9077 \mathrm{e}-6$ \\
6 & $5.9988 \mathrm{e}-7$ & $1.4474 \mathrm{e}-12$ & $2.1835 \mathrm{e}-7$ \\
7 & $3.1942 \mathrm{e}-8$ & $8.5910 \mathrm{e}-14$ & $1.2547 \mathrm{e}-8$ \\
\hline \hline
\end{tabular}

Table 3 Comparison of eigenvalues-moderate change

\begin{tabular}{ccccc}
\hline \hline $\begin{array}{c}\text { Mode } \\
\text { no. }\end{array}$ & $\lambda_{i}^{0}$ & $\begin{array}{c}\text { Fox and Kapoor's } \\
\lambda_{i}^{1}(\text { iter. 0) }\end{array}$ & $\lambda_{i}^{1}$ (iter 3) & Exact $\lambda_{i}^{1}$ \\
\hline \hline 1 & $6.8007 \mathrm{e}+04$ & $9.7977 \mathrm{e}+04$ & $9.0064 \mathrm{e}+04$ & $9.0049 \mathrm{e}+04$ \\
2 & $2.6735 \mathrm{e}+06$ & $2.9831 \mathrm{e}+06$ & $2.8705 \mathrm{e}+06$ & $2.8705 \mathrm{e}+06$ \\
3 & $2.1091 \mathrm{e}+07$ & $2.1859 \mathrm{e}+07$ & $2.1091 \mathrm{e}+07$ & $2.1090 \mathrm{e}+07$ \\
4 & $8.2307 \mathrm{e}+07$ & $8.3195 \mathrm{e}+07$ & $7.8556 \mathrm{e}+07$ & $7.8547 \mathrm{e}+07$ \\
5 & $2.2674 \mathrm{e}+08$ & $2.2991 \mathrm{e}+08$ & $2.1670 \mathrm{e}+08$ & $2.1669 \mathrm{e}+08$ \\
6 & $6.2576 \mathrm{e}+08$ & $6.2836 \mathrm{e}+08$ & $6.0003 \mathrm{e}+08$ & $5.9991 \mathrm{e}+08$ \\
7 & $1.3384 \mathrm{e}+09$ & $1.3149 \mathrm{e}+09$ & $1.2824 \mathrm{e}+09$ & $1.2824 \mathrm{e}+09$ \\
8 & $2.8149 \mathrm{e}+09$ & $2.6987 \mathrm{e}+09$ & $2.6743 \mathrm{e}+09$ & $2.6742 \mathrm{e}+09$ \\
9 & $5.6807 \mathrm{e}+09$ & $5.1008 \mathrm{e}+09$ & $5.0917 \mathrm{e}+09$ & $5.0917 \mathrm{e}+09$ \\
10 & $1.2293 \mathrm{e}+10$ & $1.0553 \mathrm{e}+10$ & $1.0406 \mathrm{e}+10$ & $1.0406 \mathrm{e}+10$ \\
\hline \hline
\end{tabular}

Table 4 Comparison of eigenvectors-moderate change

\begin{tabular}{cccc}
\hline \hline$\left\{\phi^{0}\right\}_{6}$ & Fox and Kapoor's $\left\{\phi^{1}\right\}_{6}$ & $\left\{\phi^{1}\right\}_{6}$ (iter. 3) & Exact $\left\{\phi^{1}\right\}_{6}$ \\
\hline \hline-3.9201 & -4.8353 & -4.4618 & -4.4625 \\
-0.1392 & -0.0804 & -0.0863 & -0.0862 \\
1.5953 & 3.7807 & 3.4092 & 3.4110 \\
0.1631 & 0.1603 & 0.1530 & 0.1529 \\
0.7704 & -0.8367 & -0.7196 & -0.7227 \\
-0.1675 & -0.1885 & -0.1868 & -0.1868 \\
-2.8386 & -2.3515 & -2.4258 & -2.4221 \\
0.1452 & 0.1715 & 0.1711 & 0.1712 \\
10.459 & 11.347 & 11.443 & 11.436 \\
-0.2027 & -0.2296 & -0.2312 & -0.2312 \\
\hline \hline
\end{tabular}

Table 5 Convergence of iteration-large change

\begin{tabular}{|c|c|c|c|}
\hline Iter. no. & $\begin{array}{c}\text { Average } \\
\mid \% \text { error } \mid \text { in } \lambda_{i}\end{array}$ & $\begin{array}{c}\text { Average } \\
{\left[D^{1}\right]_{i} \text { cond no. }}\end{array}$ & $\begin{array}{c}\text { Average } \\
\|\left\{\phi^{1}\right\}_{i} \text { error } \|_{2}\end{array}$ \\
\hline 0 & 76.3080 Fox & $2.0894 \mathrm{e}-05$ & 4.1988 \\
\hline 1 & 73.5823 & $1.7628 \mathrm{e}-05$ & 11.0364 \\
\hline 2 & 19.7245 & $5.9050 \mathrm{e}-06$ & 15.1258 \\
\hline 3 & 5.3836 & $3.1959 \mathrm{e}-06$ & 3.5353 \\
\hline 4 & 4.9042 & $2.4621 \mathrm{e}-06$ & 1.9003 \\
\hline 5 & 3.5970 & $1.5109 \mathrm{e}-06$ & 2.9056 \\
\hline 6 & 4.8248 & $1.6587 \mathrm{e}-06$ & 1.4242 \\
\hline 7 & 3.4145 & $1.0459 \mathrm{e}-06$ & 2.6031 \\
\hline
\end{tabular}

Averaging the percent errors for Fox and Kapoor's $\lambda_{i}^{1}$ (iter. 0 ) and $\lambda_{i}^{1}$ (iter. 3) in Table 3 gives rise to the $3.8 \%$ and $0.0055 \%$ figures in Table 2 .

In Table 4, the baseline mode shape, the perturbed mode shape from Fox and Kapoor's modal method, the perturbed mode shape at iteration 3 , and the exact perturbed mode shape are shown for the sixth eigenvector, since it was consistently 
Table 6 Severe design variable scaling sets

\begin{tabular}{|c|c|c|c|c|c|c|c|}
\hline Set & Magnitude, $\%$ & DV $1, \%$ & DV2, \% & DV3, $\%$ & DV4, $\%$ & DV5, \% & $\begin{array}{l}\text { Nonconv. } \\
\text { eigenpairs }\end{array}$ \\
\hline 6 & \pm 45 & +45 & -44 & +43 & -44.4 & +44.7 & 5 th \\
\hline 7 & \pm 50 & +49 & -48.5 & +49.4 & -48.4 & +50 & 5 th \\
\hline 8 & \pm 45 & +45 & +44 & +43 & -44.4 & -44.7 & 9th, 10th \\
\hline 9 & \pm 50 & +49 & +48.5 & +49.4 & -48.4 & -50 & 9 th, 10th \\
\hline 10 & $\ldots a$ & 0 & 0 & +40 & 0 & 0 & none \\
\hline 11 & $\longrightarrow$ & 0 & 0 & +60 & 0 & 0 & none \\
\hline 12 & - & 0 & 0 & +80 & 0 & 0 & 10th \\
\hline
\end{tabular}

${ }^{a}$ Does not apply.

the most poorly behaved eigenvector for this design variable scaling set $(0.0091$ vector norm error in iteration 3 estimate; average vector norm error for the 10 vectors was 0.0015 ). Nodal displacements and rotations alternate in the eigenvector component sequence.

It is evident that the perturbed mode shape estimate at iteration 3 is much improved over the mode shape from Fox and Kapoor's modal method, even for the most slowly converging eigenvector.

For larger design variable perturbations, convergent results (not tabulated) were obtained, but not as quickly. For design variable scaling set 2 , six iterations were necessary to converge from a $13 \%$ average error in $\lambda_{i}^{1}$ (Fox and Kapoor's modal method), a $1.0 \mathrm{e}-05$ average condition number in $\left[D^{1}\right]_{i}$, and a 3.4 average column norm of $\left\{\phi^{1}\right\}_{i}$ error to roughly the accuracy of iteration 3 earlier. For the larger perturbations of design variable scaling set 3 , eight iterations were necessary to converge from a $24 \%$ average error in $\lambda_{i}^{1}$ (Fox and Kapoor's modal method), a 1.4e-05 average condition number, and a 2.6 average column norm of $\left\{\phi^{1}\right\}_{i}$ error to the accuracy of iteration 3 earlier. For design variable scaling set 4, oscillations in the iterations began to appear, but the convergence criteria still improved from $35 \%$ (Fox and Kapoor), 1.9e-05, and 3.1 to $0.046 \%, 4.9 \mathrm{e}-08$, and 0.021 , respectively, in nine iterations. Lastly, design variable scaling set 5 produced the iteration history shown in Table 5 .

Here, the oscillations mentioned earlier are very evident, indicating that convergence is less stable for very large change. Although it appears that the algorithm is slowly converging, the averaging of the convergence criteria hides the fact that the fifth eigenpair would not converge. The other nine eigenpairs converged very quickly. The oscillations have resulted from the fifth eigenpair alone.

This nonconvergence of the fifth eigenpair for the large change case motivates the following study to determine the limitations of the method. Table 6 shows seven severe design variable scaling sets, chosen to determine where and perhaps why the method fails. It would appear that the $(+,-,+,-,+)$ pattern of severe design variable scalings apparent in DV scaling sets 5,6 , and 7 gives rise to the nonconvergence of the fifth eigenpair. Although this is not fully understood at this time, it is believed that a set of large design variable scalings with alternating signs causes an extremely large mode perturbation in the $n$th mode of an $n$ element cantilever beam. That is, prescribing large alternating design variable scalings in an eight-element cantilever beam would cause a very large mode perturbation in the eighth mode. Similarly, the $(+,+,+,-,-)$ pattern of severe design variable scalings apparent in DV scaling sets 8 and 9 give rise to the nonconvergence of the two highest eigenpairs. This is believed to occur because the stiffening of the inboard and weakening of the outboard portions of the cantilever forces the mode oscillations toward the cantilever tip. This causes a very large mode perturbation in the higher modes (e.g., the zero crossings of mode No. 10 are forced toward the tip whereas they were more evenly spaced in the baseline design). Finally, design variable scaling sets 10 , 11 , and 12 test convergence for a single severe design variable perturbation. Convergence of all 10 eigenpairs is evident for a
$60 \%$ increase in a single beam element height. It can be inferred that a large perturbation in a single element is much less severe than large perturbations on all elements.

The aforementioned perturbations are very severe because the element moments of inertia (and therefore the stiffnesses) change as the cube of the beam height, e.g., a $26 \%$ increase in beam height increases the bending stiffness by $100 \%$. The proposed perturbation method can converge for such large change, whereas higher order sensitivities based on Taylor series will diverge. When a positive $100 \%$ change is made in a Taylor series based method, the radius of convergence also includes a negative $100 \%$ change, which causes divergence. ${ }^{14}$

The only current limitation to the method is its speed because many matrix decompositions must be performed. That is, $\left[D^{1}\right]_{i}$ must be decomposed once per desired eigenvector change per iteration. This could be prohibitively costly for large problems desiring large changes in many eigenpairs. An alternative to repeated decomposition is the Sherman-Morrison formula: $\left(\left[A^{0}\right]+[\Delta A]\right)^{-1} \cong$ function of $\left[A^{0}\right]^{-1}$ and $[\triangle A]$. This will have limited use since the Sherman-Morrison formula is really a matrix generalization of a Taylor series expansion, and therefore would be limited by its radius of convergence.

\section{Conclusions}

A robust solution of the full nonlinear eigenproblem perturbation equations has been developed. No terms in the equations for $\Delta \lambda_{i}$ and $\{\Delta \phi\}_{i}$ have been neglected, and the frequency and mode shape perturbation equations are coupled (unlike the first-order system). That is, the finite change in eigenvalue is dependent on the perturbed value of the eigenvector, and the finite change in the eigenvector is dependent on the perturbed value of the eigenvalue. An iterative solution is therefore required. The familiar singularity in the solution for the eigenvector change is treated using the authors' modification of Nelson's method for resolution of the singular matrix equations. The normalization task necessary for $\{\Delta \phi\}$ also retains all terms, leading to a cubic equation for a weighting factor $c_{i}$.

In a cantilever beam example, convergence to the exact answers for eigenvalue and eigenvector changes has been shown to be achievable for very large perturbations in design variables. Fast convergence has been shown for $100 \%$ increases in stiffness and near convergence has been shown for $170 \%$ increases in stiffness, for the case of simultaneous design variable perturbations. For the case of a single design variable perturbation, convergence of all 10 eigenpairs has been shown for a $310 \%$ increase in stiffness for the perturbed beam element. This makes the proposed scheme especially valuable in the case when destruction of a component of a large structure is a possibility.

\section{Acknowledgments}

This research was funded by a Ford Motor Company Grant. The authors appreciate discussions with Ki-Ook Kim of Inha University, Inchon, Korea, on the topic of multiple eigenvalues. 


\section{References}

${ }^{1}$ Fox, R. L., and Kapoor, M. P., "Rates of Change of Eigenvalues and Eigenvectors," AIAA Journal, Vol. 6, No. 12, 1968, pp. 2426-2429.

${ }^{2}$ Sutter, T. R., Camarda, C. J., Walsh, J. L., and Adelman, H. M., "Comparison of Several Methods for Calculating Vibration Mode Shape Derivatives," AIAA Journal, Vol. 26, No. 12, 1988, pp. 1506-1511.

3Wang, B. P., "Improved Approximate Methods for Computing Eigenvector Derivatives in Structural Dynamics," AIAA Journal, Vol. 29, No. 6, 1991, pp. 1018-1020.

${ }^{4}$ Nelson, R. B., "Simplified Calculation of Eigenvector Derivatives," AIAA Journal, Vol. 14, No. 9, 1976, pp. 1201-1205.

${ }^{5}$ High, G. D., "An Iterative Method for Eigenvector Derivatives," Proceedings of 1990 MSC World Users Conference, Vol. 1, MacNealSchwendler Corp., Los Angeles, CA, March 28-30, 1990 (Paper 17).

'Eldred, M. S., Lerner, P. B., and Anderson, W. J., "Improvement of Normalization Methods for Eigenvector Derivatives," $A I A A$ Journal, Vol. 30, No. 6, 1992, pp. 1609-1616.

${ }^{7}$ Adelman, H. M., and Haftka, R. T., "Sensitivity Analysis of Dis- crete Structural Systems," AIAA Journal, Vol. 24, No. 5, 1986, pp. 823-832.

${ }^{8} \mathrm{Kim}, \mathrm{K}$. O., Anderson, W. J., and Sandstrom, R. E., "Nonlinear Inverse Perturbation Method in Dynamic Analysis," AIAA Journal, Vol. 21, No. 9, 1983, pp. 1310-1316.

${ }^{9}$ Hoff, C. J., Bernitsas, M. M., Sandstrom, R. E., and Anderson, W. J., "Inverse Perturbation Method for Structural Redesign with Frequency and Mode Shape Constraints," AIAA Journal, Vol. 22, No. 9,1984 , pp. 1304-1309.

${ }^{10} \mathrm{Kim}, \mathrm{K}$. O., "Improved Calculations of Modal Design Sensitivities," Computers \& Structures, Vol. 37, No. 5, 1990, pp. 777-780.

${ }^{11} \mathrm{Kim}, \mathrm{K}$. O., and Wallerstein, D. V., "Modal Design Sensitivities for Multiple Eigenvalues," Computers \& Structures, Vol. 29, No. 5, 1988, pp. 755-762.

${ }^{12}$ Moler, C., Herskovitz, S., Little, J., and Bangert, S., "MATLAB for Macintosh Computers: User's Guide," MathWorks, Inc., South Natick, MA, 1988.

${ }^{13}$ Whitesell, J. E., Jr., "Design Sensitivity in Mechanical Systems," Ph.D. Dissertation in Mechanical Engineering, Michigan State Univ., Lansing, MI, 1980.

\section{Introduction to Mathematical Methods in Defense Analyses}

\section{J.S. Przemieniecki}

Reflecting and amplifying the many diverse tools used in analysis of military systems and as introduced to newcomers in the armed services as well as defense researchers, this text develops mathematical methods from first principles and takes them through to application, with emphasis on engineering applicability and realworld depictions in modeling and simulation. Topics include: Scientific Methods in Military Operations; Characteristic Properties of
Weapons; Passive Targets; Deterministic Combat Models; Probabilistic Combat Models; Strategic Defense; Tactical Engagements of Heterogeneous Forces; Reliability of Operations and Systems; Target Detection; Modeling; Probability; plus numerous appendices, more than 100 references, 150 tables and figures, and 775 equations. 1990,300 pp, illus, Hardback, ISBN 0-930403-71-1, AIAA Members $\$ 47.95$, Nonmembers $\$ 61.95$, Order \#: 71-1 (830)

\section{Defense Analysis Software}

\section{J. S. Przemieniecki}

Developed for use with Introduction to Mathematical Methods in Defense Analyses, Defense Analysis Software is a compilation of 76 subroutines for desktop computer calculation of numerical values or tables from within the text. The subroutines can be linked to generate extensive programs. Many subroutines can also be used in other applications. Each subroutine fully references the corresponding equation from the text. Written in BASIC; fully tested; $100 \mathrm{~KB}$ needed for the 76 files. 1991, $131 \mathrm{pp}$ workbook, 3.5" and 5.25" disks, ISBN 0-930403-91-6, \$29.95, Order \#: 91-6 (830)

Place your order today! Call 1-800/682-AIAA

\section{CAMA:}

